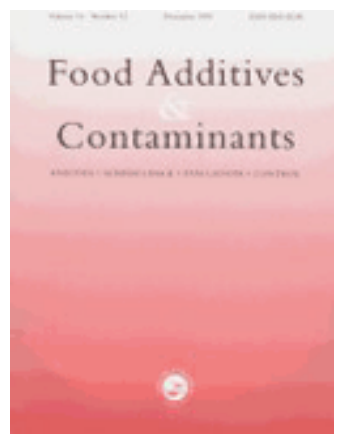

\title{
Validation of a Five Plate Test, the STAR protocol, for the screening of antibiotic residues in muscle from different animal species according to the European decision 2002/657/EC
}

\begin{tabular}{|c|c|}
\hline Journal: & Food Additives and Contaminants \\
\hline Manuscript ID: & TFAC-2009-268.R1 \\
\hline Manuscript Type: & Original Research Paper \\
\hline $\begin{array}{l}\text { Date Submitted by the } \\
\text { Author: }\end{array}$ & 02-Feb-2010 \\
\hline Complete List of Authors: & $\begin{array}{l}\text { Gaudin, Valerie; AFSSA, LERMVD } \\
\text { Hedou, Celine; AFSSA, LERMVD } \\
\text { Rault, Annie; AFSSA, LERMVD } \\
\text { Verdon, Eric; AFSSA, LERMVD }\end{array}$ \\
\hline Methods/Techniques: & In-house validation, Screening - microbial screening \\
\hline Additives/Contaminants: & $\begin{array}{l}\text { Residues, Veterinary drug residues - antibiotics, Veterinary drug } \\
\text { residues - antimicrobials, Veterinary drugs }\end{array}$ \\
\hline Food Types: & Animal products - meat, Meat \\
\hline
\end{tabular}

\section{SCHOLARONE ${ }^{\text {M }}$ Manuscripts}


1 Validation of a Five Plate Test, the STAR protocol, for the screening 2 of antibiotic residues in muscle from different animal species 3 according to the European decision 2002/657/EC 4 5 6

7 Authors : Valérie GAUDIN*, Céline HEDOU, Annie RAULT, Eric VERDON 8 9

10 Community Reference Laboratory

11 AFSSA Fougères - LERMVD

12 La Haute Marche - BP 90203

1335302 FOUGERE Cedex

14 France 15

16 Telephone : 33299947878

17 Fax : 33299947880

18 E-mail:v.gaudin@fougères.afssa.fr 19 20 21 22 


\section{ABSTRACT}

24 The STAR protocol is a Five Plate Test (FPT) developed several years ago at the 25 Community Reference Laboratory $(C R L)$ for the screening of antimicrobial residues in milk 26 and muscle. This paper presents the validation of this method according to the European 27 Decision 2002/657/EC and to an internal guideline for validation. A validation protocol based 28 on "simulated tissues" and on a list of 16 representative antimicrobials to be validated has 29 been implemented in our laboratory during several months for the STAR protocol. The 30 performance characteristics of the method have been determined (specificity, detection 31 capabilities CC $\beta$, applicability, ruggedness). In conclusion, the STAR protocol is applicable to 32 the broad spectrum detection of antibiotic residues in muscles of different animal species 33 (pig, cattle, sheep, poultry). The method has good specificity (false positive rate 4\%). The 34 detection capabilities have been determined for 16 antibiotics from different families in relation to their respective Maximum Residue Limit (MRL): beta-lactams (penicillins and cephalosporins $\leq \mathrm{MRL}$ ), tetracyclines ( $\mathrm{MRL}$ and $\leq 2.5 \mathrm{MRL}$ ), macrolides (2 $\mathrm{MRL})$, 37 quinolones $(\leq 2 \mathrm{MRL})$, some sulphonamides ( $\leq 3 \mathrm{MRL}$ ), trimethoprim (2MRL). However, the 38 sensitivity of the STAR protocol towards aminoglycosides (> $8 \mathrm{MRL}$ ) and florfenicol ( $\leq 10$ $39 \mathrm{MRL}$ ) was unsatisfactory (>> MRL). The two objectives of this study have been met: firstly to 40 validate the STAR protocol according to the European decision 2002/657/EC, then demonstrate that the validation guideline (Anonymous 2010) developed to implement this decision is applicable to microbiological plate tests even for muscle. The use of "simulated tissue appeared as a good compromise between spiked discs with antibiotic solutions and incurred tissues. In addition, the choice of a list of representative antibiotics allowed the reduction of the scope of the validation, which was already costly in time and effort.

Keywords: Validation, decision 2002/657/EC, STAR protocol, screening, antimicrobial 50 residues, muscle, plate test 


\section{INTRODUCTION}

Due to preventive or curative treatment of livestock, the presence of antibiotic residues could be found in food of animal origin. Traces of these antibiotics could cause various problems: problems of technological processing (e.g. milk), allergies to antibiotics following the ingestion of contaminated food (e.g. penicillins), antibiotic resistance of bacteria in humans, which could be transferred to pathogenic bacteria for humans. So, Maximum Residue Limits (MRLs) have been set for the antibiotics approved for use in veterinary medicine for livestock. The European Regulation No. 470/2009 of 6 May 2009 (EC 2009) establishes procedures for the establishment of Maximum Residue Limits of pharmacologically active substances in foodstuffs of animal origin and repeals Regulation No 2377/90 of 26 June 1990 (EC 1990). To monitor the presence of residues, the first step is the screening step which is to conclude whether a sample contains or not antibiotic residues at or above the MRL, in the case of permitted substances. Then, in case of positive screening it is necessary to use physico-chemical methods for the confirmation of identity and the quantification of the substance (EC 2002). The screening step is often based on microbiological screening methods that are cheap, easy to perform and do not need specific and expensive equipment. These methods are used since many years. Some of them have been developed even before the establishment of the Maximum Residue Limits (EC 1990) and many of them before the implementation of the rules for the validation of analytical methods (EC 2002).

The STAR protocol (for Screening Test for Antibiotic Residues), developed at the Community Reference Laboratory for antimicrobial residues in food (AFSSA Fougeres, France), is intended for the qualitative detection of residues of substances with antimicrobial activity in milk and muscle, using bacterial strains sensitive to antibiotics. This method is based on 5 different plates (Five Plate Test), dedicated to the detection of specific families of antibiotics. The first validation of the STAR protocol was organised in 1999 by the way of a collaborative study with spiked discs, blank muscles and incurred muscles (Fuselier et al. 2000). Seven antibiotics from 6 families have been tested. The detection levels were included between 1 $M R L$ and $4 M R L$, but it was dependent on the production of incurred materials. These results were promising for the implementation of the STAR protocol. Then the STAR protocol was validated for the screening of antibiotics in milk (Gaudin et al. 2004). The sensitivity was established by the analysis of milk samples spiked with 66 antibiotics at eight different concentrations. Ten different groups of antibiotics were studied: macrolides, aminoglycosides, cephalosporins, penicillins, quinolones, tetracyclines, sulphonamides, lincosamides, phenicols and miscellaneous drugs. The STAR protocol was able to detect 21 
89 antibiotics at or below the maximum residue limit (MRL), and a further 27 drugs could be 90 detected at levels from the MRL up to four times the MRL. The STAR protocol was at least 91 twice as sensitive as conventional methods for macrolides, quinolones and tetracyclines. 92 Each plate was preferentially sensitive for one or two families of antibacterials: the plate 93 Bacillus cereus for tetracyclines, the plate Escherichia coli for quinolones, the plate Bacillus 94 subtilis for aminoglycosides, the plate Kocuria rhizophila for macrolides and the plate Bacillus 95 stearothermophilus for sulphonamides and beta-lactams. 96

Since 2002, every analytical method used for the analysis of monitoring routine samples has to be validated according to the European Decision 2002/657/EC (EC 2002) which concerns the performance of analytical methods and the interpretation of results. The level of validation of confirmatory methods is now quite satisfactory. However, very little information is contained in that decision concerning the validation of screening methods. Two main information are contained in the decision (EC 2002): firstly which performance characteristics have to be determined for a screening method and secondly the following information about the detection capability required for screening methods. Screening methods are "only those analytical techniques, for which it can be demonstrated in a documented traceable manner that they are validated and have a false compliant rate of $<5 \%$ ( $\beta$-error) at the level of interest shall be used for screening purposes in conformity with Directive 96/23/EC (EC 1996). In the case of a suspected non-compliant result, this result shall be confirmed by a confirmatory method." Finally the difficulty is how to use this information to implement a validation for a screening method.

Moreover, the validation of microbiological screening methods presents specific issues, different from physico-chemical methods which are often specific methods. Firstly, the number of antibiotics to be validated is very high because all the antibiotics having a MRL in the corresponding matrix should be validated. It could be also the case for newly developed multi-residue methods by LC-MS/MS. However microbiological methods do not allow to identify the antibiotic residue present in the sample. So each antibiotic has to be tested independently and the number of analyses increased. Secondly, these kind of methods are only qualitative methods, giving a response as negative, positive or doubtful. Deciding on a sample size for qualitative inquiry can be even more difficult than quantitative because there are no definite rules to be followed. In general, sample size depends on the nature of the analysis to be performed, the desired precision of the estimates one wishes to achieve. The larger your sample size, the more sure you can be that their answers truly reflect the population. This indicates that for a given confidence level (e.g.. $95 \%$; $\beta$ error $=5 \%$ ), the larger your sample size $(n)$, the smaller your confidence interval (interval estimate of a 
126 population parameter). For $n=20$ and a percentage of answers of $50 \%$ (i.e. $50 \%$ answers 127 positive; $50 \%$ answers negative), the confidence interval is equal to $22 \%$. For $n=60$ and a 128 percentage of answers of 50\% (50\% positive; $50 \%$ negative), the confidence interval is 129 equal to $13 \%$. A greater sample is then required to decrease the confidence interval and 130 therefore chance of error. Therefore from a statistical point of view, the number of samples to 131 be analysed should be higher than for quantitative methods. Thirdly, spiking liquid matrices 132 (e.g. milk, juice meat) is easy and validation could be performed on spiked samples. The 133 problem is specific of solid matrices like muscle. In fact, plate tests are based on the analysis 134 of raw muscle (slices of meat). Therefore the validation should be conducted on blank and 135 incurred raw muscle. However, the production of incurred materials for each antimicrobials at 136 one concentration would be time and money consuming. As a consequence, very few 137 microbiological methods have been validated in the matrix, especially in muscle and other 138 solid matrices according to the decision 2002/657/EC (EC 2002). A new guideline document 139 supplements Commission Decision 2002/657/EC regarding the validation of screening 140 methods. The Community Reference Laboratory (CRL) in Fougères in collaboration with the $141 \mathrm{CRL}$ in Berlin and in agreement with the CRL in Bilthoven, and after consultation through the 142 NRL (National Reference Laboratories) network, has drafted this document with the purpose 143 to assist residue laboratories to validate screening methods. This document is now finalised 144 and officially published on the DGSANCO website since the $21^{\text {st }}$ of January 2010 145 (Anonymous 2010). This guideline deals with the initial validation and also a shortened or 146 'abridged' validation, which under certain conditions, allows for the transfer of methods 147 already validated in one laboratory to a second one. The guideline proposes some 148 recommendations to implement a validation protocol for screening methods. It explains the 149 performance characteristics to be determined (specificity, detection capability, robustness, $150 \quad \ldots$ ) and how to determine them in practice. Moreover, the number of samples necessary to 151 validate a screening method is discussed. Finally, some new concepts have been introduced 152 in the guideline: the preparation of "simulated tissues" and a list of representative substances 153 to be validated. These two concepts will be detailed below in the validation protocol.

154

155 The validation conducted in this study is based on this validation guideline which is also the 156 internal guideline in our laboratory for the validation of screening methods. Two objectives 157 have been set: firstly to validate the STAR protocol for its application to the detection of 158 antibiotic residues in muscles from different animal species according to the decision 159 2002/657/EC (EC 2002); secondly to show that the European guideline for the validation 160 (Anonymous 2010) and based on the decision 2002/657/EC (EC 2002) was applicable to the 161 validation of a microbiological screening method. 
162 This paper will present the validation protocol and then the results of the validation of the

163 STAR protocol for the screening of antibiotic residues in muscles of different animal species.

164

\section{MATERIAL AND METHODS}

\section{Chemicals and standard solutions}

167 Antibiotic and sulphonamide standards were provided by Sigma, except cefquinome 168 (Intervet), ceftiofur (Upjohn), enrofloxacin (Bayer), doxycycline (Virbac).

\section{STAR protocol}

171 The STAR protocol is a Five Plate Test which was already published for the analysis of milk

172 (Gaudin et al. 2004). Five test organisms (Bacillus subtilis B.G.A spores (MERCK), Kocuria 173 rhizophila ATCC 9341 (Pasteur Institute, France), Bacillus cereus ATCC 11778 (Pasteur 174 Institute, France), Escherichia coli ATCC 11303 (Pasteur Institute, France), Bacillus 175 stearothermophilus ATCC 10149 (MERCK)) were inoculated in 5 different media. The 5 176 following culture media were used respectively: Antibiotic medium II at pH 8.0 (plate Bs8), 177 Test agar at pH 8 (MERCK) (plate Kv8), Test agar at pH 6 (MERCK) (plate Bc6), Test agar 178 at pH 8 (MERCK) (plate Ec8) and Diagnostic Sensitive Test (DST) (OXOID commercialised 179 by UNIPATH LTD, Basingstoke, Hampshire, UK) (plate Bst). Culture media were prepared 180 as recommended by the supplier and sterilised. Then $5 \mathrm{ml}$ of inoculated medium were added 181 on a Petri dish placed on a cold horizontal surface. In routine use, a cylindrical plug of $8 \mathrm{~mm}$ 182 in diameter and $2 \mathrm{~cm}$ long is cut in frozen muscle using a cork borer. Then, slices of muscle 183 samples of $2 \mathrm{~mm}$ in thickness are cut and put on the plates. The same protocol was applied 184 to "simulated tissues". Finally the plates are incubated: at $30^{\circ} \mathrm{C}$ for at least 18 hours for Bs8 185 and $\mathrm{Bc} 6$, at $37^{\circ} \mathrm{C}$ for at least 24 hours for $\mathrm{Kv} 8$, at $37^{\circ} \mathrm{C}$ for at least 18 hours for $\mathrm{Bc} 6$ and at $18655^{\circ} \mathrm{C}$ for 15 to 16 hours for Bst.

188 A muscle sample was considered positive when the inhibition zone around meat sample was 189 equal or superior to $2 \mathrm{~mm}$ in width on plates Bs 8, Kv 8, Bc 6 and Ec 8 and / or the inhibition 190 zone equal or superior to $4 \mathrm{~mm}$ in width on plate Bst.

192 Since 2004, medium test agar $\mathrm{pH} 7.2$ has been replaced by antibiotic medium II at $\mathrm{pH} 8.0$ 193 (Difco, reference 259310) because it was not commercialised anymore.. Moreover, the 194 positive control of plate Bst was modified for practical reasons: sulfamethazine at $1000 \mathrm{Mg} \mathrm{l}^{-1}$ 195 has been replaced by amoxicillin at $40 \mu \mathrm{g} \mathrm{I}^{-1}$. Positive controls consist of $30 \mu \mathrm{l}$ of antibiotic 196 solutions which are put on paper discs of $9 \mathrm{~mm}$ diameter (Durieux, France). There is a 197 specific positive control for each plate: streptomycin at $2000 \mathrm{\mu g} \mathrm{I}^{-1}$ on plate Bs8, tylosin at $1981000 \mathrm{~g} \mathrm{I} \mathrm{I}^{-1}$ on plate Kv8, oxytetracycline at $800 \mu \mathrm{g} \mathrm{I}^{-1}$ on plate Bc6, enrofloxacin $800 \mu \mathrm{g} \mathrm{I}^{-1}$ on 
199 plate Ec8 and amoxicillin at $40 \mathrm{\mu g} \mathrm{I}^{-1}$ on plate Bst. The validity of each day of analysis 200 depends on the results of the positive controls which have to be included in the following 201 intervals: $5.5 \pm 1.5 \mathrm{~mm}, 6.5 \pm 1.5 \mathrm{~mm}, 6.0 \pm 1.5 \mathrm{~mm}, 7.0 \pm 1.5 \mathrm{~mm}$ and $6.0 \pm 1.5 \mathrm{~mm}$ for Bs8, 202 Kv8, Bc6, Ec8 and Bst respectively.

203

204

\section{Validation protocol}

205

\section{Simulated tissues}

In 2002, we have studied the sensitivity of 35 antibiotics by the way of antibiotic spiked 207 discs. However, this way of working was not completely satisfying because the interference of the muscle matrix was absent. In fact, Okerman et al (1998a) showed that the tissue matrix has an effect on the sensitivity of the test plates. During that study, pieces of frozen meat laid on paper discs impregnated with antibiotic standard solutions have been used for the validation. Usually inhibition zones decreased when spiked meat samples have been analysed, compared to antibiotic spiked discs without meat. The same conclusions were reported by Pikkemaat et al. (2007). Because of the difficulty and the high cost of production of incurred materials for validation of a microbiological plate test, it was decided to work on what we called "simulated tissue. Furthermore, it was impossible to mix several antibiotics in the same sample, since the method was not specific and did not identify the molecule that produced an inhibitory effect. Moreover, one could observe a cumulative effect of antibiotics in their inhibitory activity when several antibiotics are contained in one sample.

Therefore, a preliminary study was conducted to determine what was the best way to prepare simulated tissues and what kind of preparation would give the closest result to the actual samples. This study was based on the experience of 2 National Reference

\section{Production of spiked materials ("Simulated tissue")}

230 A preliminary study has been conducted to determine the concentrations of antibiotics to be 231 spiked in muscle samples for later validation. Sixteen different antibiotics have been tested at 232 various concentrations (the MRL and according to the assumed detection limit at half MRL, 2 233 times the MRL or more) in simulated tissues. The tested concentrations were based on the 234 results of the validation of the STAR protocol in milk (Gaudin et al. 2004). These tests were 235 repeated several times. The concentration which always gave positive results (inhibition 
236 zones higher than $2 \mathrm{~mm}$, generally between 3 and $4 \mathrm{~mm}$ ) and if possible concentrations

237 lower than or equal to the respective MRLs have been chosen.

238 The antibiotics and the corresponding concentrations chosen for the validation are presented 239 in Table 1.

Insert table 1 about here

Sixteen different antibiotics were used in this study. Nine different groups of antibiotics were 244 studied : macrolides, aminoglycosides, cephalosporins, penicillins, quinolones, tetracyclines, 245 sulphonamides, lincosamides and miscellaneous drugs. Stock solutions of the 16 different 246 antimicrobials were prepared at a concentration of $1 \mathrm{mg} \mathrm{ml}^{-1}$, after correction of potency. 247 Then working solutions were prepared by dilutions in distilled water. Different batches of 248 muscle were purchased in supermarkets. Muscle was firstly coarsely minced. To prevent 249 antibiotic contamination, finely minced blank muscle samples were prepared first, the same 250 day, with the 4 different batches of muscle, to be tested in parallel with the spiked samples. 251 Then $1 \mathrm{ml}$ of working solution was added to $100 \mathrm{~g}$ of blank minced muscle. Spiked muscle 252 was homogenised during 15 minutes in the same rotary hatcher. At the end, the meat is 253 finely minced. Each muscle material was finally put in plastic bottles or plastic bags. Each 254 material was codified by the director of the study and then was frozen at $-20^{\circ} \mathrm{C}$. The 255 "simulated tissues" were always analysed in a maximum period of 1 month.

\section{Specificity}

25849 batches of pork muscle of different origins were analyzed in the end. Most of them (40 259 batches) have been tested in blind duplicate on 2 different days and by 2 different 260 technicians.

262 Detection capabilities $\mathbf{C C} \beta$

263 According to EU Commission Decision 2002/657/EC (EC 2002), the detection capability $264(\mathrm{CC} \beta)$ of a method is defined as "the smallest content of the substance that may be 265 detected, identified and/or quantified in a sample with an error probability of $\beta$. In the case of 266 substances with an established permitted limit, this means that the detection capability is the 267 concentration at which the method is able to detect permitted limit concentrations with a 268 statistical certainty of $1-\beta .$.

270 Given the expected detection capabilities for many antibiotics, which are often close to the 271 MRLs, it was chosen to determine the detection capabilities by analyzing 60 minced muscle 
272 samples spiked at one antibiotic concentration, instead of 20 samples to reach the most 273 statically significant determination of the CC $\beta$ (EC 2002).

\section{Insert Figure 1 about here}

The preparation of the samples is detailed in figure 1. To introduce a maximum of variability in the determination of the detection capabilities, 30 samples have been prepared and analyzed by one technician and another 30 samples by other technician. In addition, the 30 280 samples have been divided into 2 sets of preparation. In each set, 15 samples for the same 281 antibiotic concentration have been prepared and analyzed by each technician. For each 282 antibiotic and each technician, the 2 sets have been spaced out, to introduce variability in the 283 period of analysis (ambient conditions) and therefore variability of batches (media, bacteria, 284 antibiotic standard...). Moreover, in each set of preparation of 4 antibiotics, 4 different 285 batches of porcine muscle have been used. Finally, on each day of preparation, 2 different 286 working solutions have been prepared from the stock solution of antibiotic and used to spike 287 the different batches of muscle. The coding of the samples has been performed by the head 288 of the study, to ensure that the analyses would be performed blindly, and then frozen at -20 $289{ }^{\circ} \mathrm{C}$.

291 In the determination of specificity and detection capabilities, all samples have been analyzed 292 on the 5 plates of the STAR protocol to check the specificity of the plates for the different 293 families of antibiotics. Five days of analyses have been performed with the STAR protocol for 294 each set of preparation and each technician. Each day, 14 samples have been analysed 295 blindly (12 spiked samples and 2 blank samples). Therefore, all blank and spiked samples 296 have been analyzed twice or 3 times, on 2 or 3 different days.

298 One wants to underline that the analysis of the 60 samples for each antimicrobial were 299 performed step by step. When one false negative result or more was obtained after the 300 analysis of 10 samples, either the validation was stopped at this concentration and started 301 again with an increased concentration if it was of interest (e.g. near the MRL) or the 302 validation went on with the same antibiotic and the same concentration because the 303 concentration was already much higher than the concerned MRL.

305 Determination of $\mathrm{CC} \beta$ : After the analysis of the 60 spiked samples, the concentration level, 306 where only less than $5 \%$ of false compliant results remains was the detection capability CC $\beta$ 307 of the method ( 3 false compliant results maximum out of 60 spiked samples). 


\section{Applicability study}

310 The STAR protocol is implemented in parallel with the Four Plate Test for the analysis of

311 muscles from different animal species , during the routine analysis of field samples (cattle,

312 sheep, poultry, ...) in National Monitoring Plans since at least 5 years. Then, the positive

313 samples at the screening step are confirmed by a multi-residue LC-MS/MS method (data to

314 be published). Therefore, we have a lot of experience about the applicability of the method to

315 muscles from different animal species.

317 However, to complete this study, we decided to perform a study to determine the CC $\beta$ of 318 some antibiotics in cattle, sheep and poultry muscles, antibiotics for which the CC $\beta$ were 319 determined previously in porcine muscle. Therefore, the CC $\beta$ of 5 antimicrobials (penicillin G 320 at $25 \mu \mathrm{g} \mathrm{kg}^{-1}$, doxycycline at $100 \mu \mathrm{g} \mathrm{kg}^{-1}$, erythromycin at $400 \mu \mathrm{g} \mathrm{kg}^{-1}$, gentamicin at $6000 \mu \mathrm{g}$ $321 \mathrm{~kg}^{-1}$, enrofloxacin at $200 \mu \mathrm{g} \mathrm{kg}^{-1}$ ) have been determined with 20 samples from each of the 3 322 species over 5 days and have been compared with the CC $\beta$ calculated for porcine muscle. In 323 the applicability study, only the specific plate (which has presented inhibition zones for pig 324 muscle) was tested with the corresponding antibiotic.

326 The applicability would be proved if the CC $\beta$ determined for these species are similar to the 327 CC $\beta$ determined for porcine muscle (the average inhibition zones obtained for each species 328 on the specific plate should be similar: accepted deviation $\pm 25 \%$ ). A variability of $25 \%$ is 329 usually accepted with microbiological methods (for positive controls or spiked muscles). 330 Moreover, this range of variability was observed during the validation in porcine muscle. The 331 specificity of the STAR protocol with other species has also been studied by the analysis of 332 different blank muscle samples.

\section{3}

\section{Ruggedness study}

335 The ruggedness of the method has been partly evaluated during the validation study, since 336 different batches of media, bacterial strains, antimicrobial standards have been used. In 337 addition, 2 technicians have been involved in the study, analysing each half of the samples 338 and the variability between technicians could be estimated.

340 In the decision 2002/657/EC (EC 2002), the ruggedness is defined as " the susceptibility of 341 an analytical method to changes in experimental conditions [...] under which the method can 342 be applied as presented or with specified minor modifications". A ruggedness study based on 343 an experimental design was carried out and thus allowed testing analytical parameters which 344 were different from those tested during the first step of validation. A full factorial design was 
345 chosen for the analysis of the effects and interactions of 4 independent factors (Renard et al. 346 1992). The design matrix is presented in Table 2.

\section{Insert Table 2 about here}

350 Factors which may influence the measurement results have been selected: bacteria 351 concentration (A), medium quantity (B) in the plate, incubation time (C) and pre-incubation 352 time at room temperature (D). These factors have been modified in an order of magnitude 353 corresponding to the usual differences: factor $A$ concentration in bacteria: $\pm 30 \%$; factor $B$ 354 quantity of medium $5 \pm 0.5 \mathrm{ml}$; factor $\mathrm{C}$ incubation time $\pm 10 \%$; factor $\mathrm{D}$ pre-incubation time 355 (period at room temperature before incubation in the incubator: 1hour of pre-incubation or no 356 pre-incubation).

The ruggedness study has been focused on 6 different representative antimicrobials which

359 were specifically detected on each of the 5 plates on muscle: penicillin $\mathrm{G}$ at $25 \mathrm{\mu g} \mathrm{kg}^{-1}$ and 360 sulfadimethoxine at $300 \mu \mathrm{g} \mathrm{kg}^{-1}$ on plate Bst, doxycycline at $100 \mu \mathrm{g} \mathrm{kg}^{-1}$ on plate Bc6, 361 erythromycin at $400 \mu \mathrm{g} \mathrm{kg}^{-1}$ on plate Kv8, gentamicin at $6000 \mathrm{\mu g} \mathrm{kg}^{-1}$ on plate Bs8, 362 enrofloxacin at $200 \mu \mathrm{g} \mathrm{kg}^{-1}$ on plate Ec8. The chosen concentrations were equal to the 363 detection capabilities determined in the first part of the validation study for each of these 364 antibiotics, except for gentamicin. The samples were prepared from pig muscle only. All 365 b$^{2}$ lank and spiked minced muscle samples were prepared the day before starting the 366 ruggedness study and were frozen at $-20^{\circ} \mathrm{C}$. The study was performed blindly (codified 367 samples). Then each day of analysis (each run), 4 different blank materials and 4 different 368 spiked materials per antibiotic were analysed.

\section{RESULTS AND DISCUSSION}

371 Specificity

372 Specificity and detection capabilities were determined for pig matrix because it is the most 373 common species analyzed in the laboratory. 49 different batches of porcine muscle have 374 been tested. Few variations have been observed. Of 176 analyses in total, only 7 gave false 375 positive results $(4 \%)$. The 7 false positive results were obtained with 5 different muscle 376 batches (2 false positive results for 2 batches and 1 false positive result for each of 3 377 batches). All false positive results have appeared on the plate Bst, none on the other plates. 378 The specificity of the STAR protocol for the detection of antimicrobial residues in porcine 379 muscle was very satisfactory. 


\section{$381 \quad$ Detection capabilities}

382 The results presented in table 3 are those obtained from 60 samples for each antibiotic at

383 least one tested concentration.

384

385

Insert Table 3 about here

386

387 For some antibiotics, after 15 or 30 analyzes by one or two technicians, either it turned out 388 that too many false negative results were obtained at the selected concentration or on the 389 opposite the first tested concentration gave higher inhibition zones than anticipated, 390 suggesting that the validation could be performed at a lower concentration. At these 391 concentrations, less than 60 samples have been analyzed, however it was helpful for the 392 determination of the detection capability.

394 Figure 2 is a graphical representation of the relationship between detection capabilities 395 calculated for the 16 representative antibiotics and their respective MRLs.

\section{Insert Figure 2 about here}

This figure allows one to quickly visualize the difference between CC $\beta$ and MRLs. The 400 respective MRLs $\left(\mu \mathrm{gg}^{-1}\right)$ are represented in plain bars. The hatched bars represent the 401 respective $\operatorname{CC} \beta\left(\mu \mathrm{g} \mathrm{kg}^{-1}\right)$. Therefore, the highest is the hatched area, the least sensitive is the STAR protocol for the corresponding antibiotic. On the opposite, when plain zone and hatched zone are very near, the sensitivity of the method for the corresponding antibiotic is satisfactory. When the CC $\beta$ is higher than $1000 \mu \mathrm{g} \mathrm{kg}^{-1}$, the value of the CC $\beta$ is written on the top of the corresponding bar.

As a conclusion, the detection capabilities of penicillin $G$, cloxacillin and doxycycline were 408 equal to or lower than their respective MRLs. The detection capabilities of tylosin, 409 sulfadimethoxine, oxytetracycline, trimethoprim, erythromycin, ceftiofur, enrofloxacin and 410 cefquinome were between 1.5 and 3 times their respective MRLs. The detection capability of 411 lincomycin was between 4 and 5 times its MRL. Finally, the detection capabilities of 412 gentamicin (GTM) (> 40*MRL), dihydrostreptomycin (DHS) (> 8*MRL) and florfenicol (> $41310^{\star} \mathrm{MRL}$ ), were much higher than their respective MRLs. The STAR protocol is not suitable 414 for the detection of the 2 aminoglycosides and probably not for the detection of the entire 415 family, given the activity profiles obtained during the validation in milk (Gaudin et al. 2004). In 416 fact, these activity profiles showed that GTM and DHS were the 2 aminoglycosides which 417 were detected at the lowest concentrations, especially GTM. The sensitivities for GTM in milk 
$418\left(\mathrm{MRL}=100 \mu \mathrm{g} \mathrm{I}^{-1}\right)$ and to DHS $\left(\mathrm{MRL}=200 \mathrm{~g} \mathrm{~g}^{\mathrm{I}^{-1}}\right)$ were equal to 3 and 5 times the respective 419 MRLs. The sensitivities determined in milk for the other aminoglycosides were as follows: 420 neomycin $1500 \mu \mathrm{g} \mathrm{I}^{-1}\left(\mathrm{MRL}=1500 \mu \mathrm{g} \mathrm{I}^{-1}\right)$, streptomycin $1000 \mu \mathrm{g} \mathrm{I}^{-1}\left(\mathrm{MRL}=200 \mu \mathrm{g} \mathrm{I}^{-1}\right)$, 421 kanamycin $1000 \mathrm{~g} \mathrm{I}^{-1}\left(\mathrm{MRL}=150 \mathrm{~g} \mathrm{I}^{-1}\right)$, spectinomycin $40000 \mathrm{~g} \mathrm{I}^{-1}\left(\mathrm{MRL}=300 \mathrm{~g} \mathrm{I}^{-1}\right)$, 422 paromomycin $2000 \mathrm{mg} \mathrm{l}^{-1}$ (not authorised in milk), apramycin $4000 \mathrm{\mu g} \mathrm{I}^{-1}$ (not authorised in 423 milk). Therefore, the sensitivities for GTM and DHS were much better in milk than in muscle. 424

425 During the determination of detection capabilities in porcine muscle, it was demonstrated that 426 the STAR protocol could provide an help to confirm the identity of a family of molecules 427 present in the sample. Table 4 shows the plates on which each antibiotic reacted 428 preferentially. Some antibiotics (e.g. cefquinome, ceftiofur, doxycycline, enrofloxacin and 429 florfenicol) caused inhibitions onto several plates at the tested concentrations (including $430 \mathrm{CC} \beta)$. 431

\section{Insert Table 4 about here} 433

434 The results were obviously similar to the global orientation given in the STAR protocol: beta435 lactams and sulphonamides were detected preferentially on plate Bst, tetracyclines on plate 436 Bc6, quinolones on plate Ec8 and macrolides on plate Kv8. However, there were 2 437 exceptions. The aminoglycosides have been detected on plate Bst with the concentrations 438 tested for the determination of $\mathrm{CC} \beta$, whereas for higher concentrations than $\mathrm{CC} \beta$, they were 439 detected on plate Bs8 = Bs7.2). However, when the validation of the STAR protocol has 440 been performed in milk, Bs8 was the specific plate for the detection of aminoglycosides. 441 Secondly, tylosin was detected preferentially on Bst instead of Kv8 at this concentration, 442 which had already been observed during the validation of the STAR protocol in milk. So if a 443 positive result is reported on plate Bst, the confirmation should be directed onto beta444 lactams, sulphonamides, but also tylosin and aminoglycosides. 445

\section{Applicability study}

447 Given the unsatisfactory results for aminoglycosides (>> MRL), an additional study was 448 conducted on GTM and DHS to choose one of these antibiotics and the good concentration 449 for applicability and ruggedness studies. This study determined that the detection capability 450 of GTM was around $4000 \mu \mathrm{g} \mathrm{kg}^{-1}$ and DHS around $6000 \mu \mathrm{g} \mathrm{kg}{ }^{-1}$. Therefore, GTM at $6000 \mu \mathrm{g}$ $451 \mathrm{~kg}^{-1}$ has been chosen, a concentration higher than the estimated detection capability (4000 $452 \mu \mathrm{g} \mathrm{kg}^{-1}$ ) due to reading difficulties (Partial Inhibition Zone (PIZ) and regrowth in the inhibition 453 zone). 
454 Figure 3 represents the average inhibition zones on the 5 different plates (Bst, Bc6, Kv8, Ec8 455 and Bs8) for blank muscles of different animal species and muscles spiked with 5 different 456 antibiotics (each one reacted specifically on one of the plates) : penicillin $\mathrm{G}$ at $25 \mathrm{\mu g} \mathrm{kg}^{-1}$ on 457 Bst (figure 3a), doxycycline at $100 \mathrm{\mu g} \mathrm{kg}^{-1}$ on Bc6 (figure 3b), erythromycin at $400 \mathrm{\mu g} \mathrm{kg}^{-1}$ on $458 \mathrm{Kv} 8$ (figure 3c), enrofloxacin of $200 \mu \mathrm{gg}^{-1}$ on Ec8 (figure 3d) and gentamicin at $6000 \mu \mathrm{kg}^{-1}$ 459 on Bs8 (figure 3e). The results for the porcine muscle were those obtained during the 460 determination of $\mathrm{CC} \beta$ for 4 plates (Bst, Bc6, Kv8, Ec8 ) and during the additional study on 461 aminoglycosides for plate Bs8.

462

463 Insert Figure 3 about here

464

465 The overall average inter-species was equal to $9.0 \pm 1.3 \mathrm{~mm}, 5.2 \pm 1.0 \mathrm{~mm}, 5.2 \pm 1.4 \mathrm{~mm}$, $466 \quad 6.1 \pm 1.1 \mathrm{~mm}$ and $5.4 \pm 0.9 \mathrm{~mm}$ for Bst, Bc6, Kv8, Ec8 and Bs8 respectively. Whichever was 467 the tested antibiotic and the plate, the discrimination between blank and spiked samples was 468 very clear and easy, for each species. Moreover, the average inhibition zone (IZ) for cattle, 469 sheep and poultry muscle was in the fixed interval of plus or minus $25 \%$ compared to the 470 average IZ for porcine muscle for plates Bst, Bc6, Ec8 and Bs8. Concerning plate Kv8, the average inhibition zone (IZ) for cattle and sheep muscle was in the fixed interval of plus or minus $25 \%$ compared to the average IZ for porcine muscle. The poultry muscle was out of 473 the interval for plate Kv8, but with an average inhibition zone of $33 \%$ higher than the porcine 474 muscle, so the sensitivity was better in the poultry muscle.

476 Two blank samples of cattle, 2 sheep and 2 poultry were analyzed for each plate and the 477 analyses were repeated for 5 days. Over 10 cattle tested, we got 2 false-positive results, 1 478 for sheep and 1 for poultry on Bst plate. The blank bovine and ovine samples gave IZ of 3.7 $479 \pm 0.7$ and $3.3 \pm 0.4 \mathrm{~mm}$, higher than porcine $(1.8 \pm 1.4 \mathrm{~mm})$ and poultry $(2.0 \pm 1.4 \mathrm{~mm})$ blank 480 samples. All blank samples gave non specific inhibition on the plate Bst, higher than for the 481 other plates. That is why the positivity threshold of the plate Bst was set at $4 \mathrm{~mm}$, while the 482 positivity threshold for the other plates was set at $2 \mathrm{~mm}$ (Fuselier at al. 2000). No false483 positive results were obtained on the plate, whatever the species, on the 4 other plates.

484 In conclusion, the STAR protocol is applicable for the screening of antibiotic residues in the 485 muscle of the 4 major species: pig, cattle, sheep, poultry and by extension the muscle of 486 minor species, whatever is the plate. 


\section{Ruggedness study}

490 During the determination of specificity and detection capabilities of the 16 antimicrobials, lots 491 of different media (different preparation and validity date), different preparations of bacteria, 492 different batches of antibiotic standards have been used over a period of 9 months of testing. 493 Looking at the quantitative results (inhibition zones) obtained by the 2 technicians on muscle 494 samples, , the variability (standard deviations) was rather low, similar to or lower than the 495 variability that is fixed for the positive controls of the method. During this period, the positive 496 controls were included in the intervals set in the STAR protocol. This is the first track to 497 conclude that the STAR method is robust. 498

499 The average inhibition zones and the standard deviations obtained during the ruggedness 500 study and during the first part of the validation (determination of CC $\beta$ ) have been compared 501 and are presented in Table 5. 502

\section{Insert Table 5 about here} 504

505 When the standard deviation in ruggedness conditions (SDi) is significantly larger than the 506 standard deviation of the method in terms of reproducibility, the conclusion is predictable that 507 all factors taken together have an impact on the results, even if no single factor has 508 significant influence. In this case, the method is not sufficiently robust regarding the range of 509 tested variations. From a quantitative point of view, we did not observe significant differences 510 between the SD in ruggedness conditions and the SD during the first part of the validation for 511 the 6 tested antibiotics. Therefore, the STAR protocol seemed to be robust for the 5 plates 512 regarding the range of tested variations. 513

514 If we look at the mean inhibition zones obtained at each day (run) during the ruggedness 515 study to the mean inhibition zones obtained during the determination of detection 516 capabilities, it seemed that the sensitivity is very near, even if the average inhibition zones 517 were often lower during the ruggedness study. In fact, the average inhibition zone of SDMX 518 on plate Bst was significantly lower during the ruggedness study (Table 5). Therefore some 519 of the tested factors had an effect on the sensitivity. Sulphonamides tested concentrations 520 often gave Partial Inhibition Zones (PIZ), which are sometimes quite tricky to read for 521 someone not trained. On the opposite, beta-lactams usually gave clear inhibition zones. So, 522 slight variations like in the ruggedness study, even for a trained people, led to more effect on 523 these PIZ than on clear inhibition zones. Therefore, it is logical that the Bst plate is less 524 robust for the detection of SDMX than for penicillin $\mathrm{G}$. 
525 The ruggedness of the STAR method has been evaluated using an experimental design. The 526 influence of the 4 factors (concentration of bacteria, quantity of medium, incubation time and 527 pre-incubation time) on the mean inhibition zone (quantitative result), repeatability (coefficient 528 of variation $\mathrm{CV} \%$ ) and false positive and false negative rate (qualitative result), as well as the 529 interactions between factors, have been evaluated (Table 2). The responses for each plate 530 for each run (day) were indicated (mean inhibition zone (IZ), the coefficient of variation (CV $531 \%)$ on the inhibition zone and false positive and false negative rates when influence was 532 observed). From these results, the exploitation of the experimental design has been 533 performed. The results are presented in Table 6 for the 6 antibiotics and the 5 plates.

\section{Insert Table 6 about here}

In conclusion, whatever the plates, there was no significant effect of analytical factors tested on the qualitative results of the STAR method (no influence on the false-positive rate and false-negative rate), except for the plate Bst with SDMX (Table 6). When testing SDMX, on day 4 , the 4 tested samples gave false-negative results (Table 7). Therefore it has a strong impact of the combination of factors tested that day: $A+, B+, C-$, D-, i.e. increasing the

542 concentration of bacteria, increasing the amount of medium, reducing the incubation period 543 and without pre-incubation. In the optimization of microbiological methods, it is well known 544 that increasing the concentration of bacteria or increasing the amount of medium may 545 decrease the sensitivity of the plate. Moreover, as seen with other plates, a decrease of the 546 incubation period can indeed reduce the sensitivity. It is also logical that a lack of pre547 incubation may decrease the sensitivity of the plate. It was observed in fact that an increase 548 of factors $A$ and $B$ may increase the false negative rate, while an increase of the incubation 549 time and pre-incubation of one hour may increase the sensitivity. It is noticeable that 1 hour 550 of pre-incubation at room temperature increased the mean inhibition zone of SDMX of $17 \%$.

\section{Insert Table 7 about here}

554 Regarding the classical variability of results of microbiological plate tests, none of the factors or combination of factors had significant effect on the mean inhibition zone (quantitative result) for any of the tested antibiotics on the 5 plates. Finally, no significant effect has been

557 observed on the repeatability of the method. Moreover, slights changes in the coefficient of 558 variation never had effect on the qualitative results of the test for the tested antibiotics.

559 Therefore, the STAR protocol is a robust method for the detection of antibiotic residues in 560 muscle. Some recommendations are given in the STAR protocol, concerning the different 561 incubation periods for the 5 plates, because incubation time was already known as a critical 
562 parameter. In the range recommended in the protocol, the method is robust. It should be 563 noticed that increasing the incubation time for plate Kv8 increased the sensitivity of the plate 564 of $13 \%$ (Table 6). This is the reason why a longer incubation time (at least 24 hours) 565 compared to the other plates is recommended in the STAR protocol. However, even at the 566 lowest incubation time (21 hours), the results were satisfactory.

568 Finally, it has been demonstrated that a pre-incubation of 1 hour at room temperature could 569 have a positive effect on the sensitivity of the test (plates Ec8, Bst (SDMX), Kv8) or no effect 570 (plates Bc6, Bs8, Bst (penicillin G)), compared to no pre-incubation. However, pre-incubation 571 is not a critical parameter because if people does not apply a pre-incubation, the results 572 would be satisfactory and if pre-incubation is performed, the results would be equal or better. 573 A recommendation of pre-incubation 1 hour at room temperature, should be added in the 574 next version of the STAR protocol. Moreover, every day of analysis, specific positive control 575 antibiotic paper discs are put on each plate. The results of these positive controls should be 576 included in the range given in the STAR protocol. If it is the case, the results are valid.

\section{Discussion}

579 At this time, very few laboratories tried to validate their screening methods (microbiological or 580 immunological methods) according to the decision 2002/657/EC (EC 2002) for 2 main 581 reasons. Firstly, the decision 2002/657/EC (EC 2002) gave very little information and no 582 technical recommendations for the implementation of the validation of a screening method. 583 Secondly, especially for the validation of microbiological plate tests, the validation requires a 584 long work, is time-consuming and quite expensive. In our laboratory, which is the Community 585 Reference Laboratory for antibiotic residues, the validation of screening methods has been a 586 subject of interest for many years. At this time, the validation of immunological tests and 587 microbiological tests (tube tests and plate test) have been performed in our laboratory 588 according to the European decision 2002/657/EC (Gaudin et al. 2004; Gaudin et al. 2007; 589 Gaudin et al. 2009a; Gaudin et al. 2009b). The former "detection limit" has been replaced by 590 a new performance characteristic called "detection capability CC $\beta$ ". To our knowledge, the 591 other validation studies of microbiological plate tests according to the European decision (EC 592 2002) were from a National Reference Laboratory in the Netherlands (Pikkemaat et al. 2007; 593 Pikkemaat et al. 2008; Pikkemaat et al. 2009b).

595 Regarding the time needed for this validation study, it would have been impossible to 596 validate the STAR protocol for all the antibiotics having a MRL in muscle matrix (more than 59750 antibiotics). The work of validation would be too long, expensive and laborious. Therefore 
598 the recommendation to use a list of representative antibiotics was a very good compromise 599 and allowed to reduce drastically the scope of the validation. It has been proposed since the 600 first version of the European guideline for the validation of screening method in 2005 to 601 validate a wide range test only for a list of representative antibiotics. It was adopted in the 602 final version of the guideline (Anonymous 2010). The choice of the representative antibiotics 603 is not fixed and is dependant of different factors: the activity patterns of different antibiotics in 604 one family, the matrix, the use of antibiotics in one specific country, the assumed sensitivity 605 of the method towards some antibiotics. The first step is to conduct a preliminary study that 606 should allow to determine a common pattern of activity for one family or at least several 607 substances of the family on a specific class of bacteria. Therefore one compound could be 608 chosen to be representative of the other substances of the family in term of activity profile on 609 bacteria. Then different antibiotics should be chosen for example for milk or muscle matrices. 610 In fact the antibiotics used for intra-mammary treatment or for oral use could be different. 611 Therefore, the interest of one antibiotic only used as intra-mammary treatment is very limited 612 for a validation in muscle. Furthermore, if some antimicrobials are not used or not registered 613 in some countries, there is no interest to validate for this compound if the method is intended 614 to a national control. Finally, the selected analytes are dependent of the analytical method. 615 So if the method to validate clearly badly detect one of antimicrobial, it is needless to 616 determine its detection capability because this antibiotic would not be included in the scope 617 of the method.

619 A similar proposition of validating for a list of representative antibiotics was made by 620 Pikkemaat et al. (2009b). After the determination of the activity profiles of 36 antibiotics for 621 the NAT-post-screening test for the detection of antibiotic residues in kidney, the authors 622 suggested that the validation could be performed on a list of representative antibiotics to 623 reduce the scope of validation for routine field laboratories for example.

625 One list of representative antibiotic was initially included as an example in the guideline 626 which was extracted from the validation of the STAR protocol in milk (Gaudin et al. 2004). In 627 fact, activity patterns have been determined for 66 antimicrobials having MRL in milk. The 628 conclusion was that several antibiotics in one family could be gathered into one group 629 because they showed similar activity profiles (same specific plate, similar sensitivity). Each 630 family could be divided into 2 or more groups. Finally, 1 or 2 antibiotics per family have been 631 chosen because its (or their) activity pattern (s) was (were) representative of one (or 2) 632 group(s) of antimicrobials in the same family. Compared to this list of representative 633 antibiotics, some molecules were replaced by others during the validation study of the STAR 634 protocol, for the following reasons: 
635 - Some antibiotics are only used as intra-mammary treatment (e.g. cefalonium has been replaced by ceftiofur because ceftiofur is widely used in cattle and swine to treat respiratory diseases while cefalonium is only administered intra-mammary), Moreover, it was determined during the validation of the STAR protocol in milk (Gaudin et al. 2004) that the least detected cephalosporins were cefquinome (MRL = 640 641 $\left.50 \mu \mathrm{g} \mathrm{I}^{-1}\right)$ and cefalexin $\left(\mathrm{MRL}=200 \mu \mathrm{g} \mathrm{I}^{-1}\right)$. On the opposite, the best detected cephalosporins were cefazolin (Annex II for all tissues except milk) and cephapirin $\left(\mathrm{MRL}=50 \mu \mathrm{g} \mathrm{I}^{-1}\right)$. Finally, ceftiofur $\left(\mathrm{MRL}=1000 \mu \mathrm{g} \mathrm{I}^{-1}\right)$ was better detected than cefquinome but less detected than cefazolin. Therefore, we have chosen to validate for ceftiofur and cefquinome because one was the least detected antibiotic of the family, with a low MRL (cefquinome) and the other (ceftiofur) has an intermediary detection with a high MRL. Regarding the activity profiles on the STAR protocol, it was assumed that if cefquinome is detected at $2000 \mathrm{\mu g} \mathrm{kg}^{-1}$ (CCbeta), cefazolin and cephapirin would be easily detected.

- The antibiotic should be largely used in the country of implementation of the method (e.g. in France, sulfamethazine replaced sulfathiazole). Moreover, colistin which belongs to the polymyxin family has been replaced by a second macrolide: tylosin because macrolides are often used for animal treatment (cattle, swine, poultry).

- During the development of the STAR protocol, it was already shown that the method had a very poor sensitivity for some antibiotics (e.g. flumequine, sulfaguanidine, colistin and spectinomycin), largely above the respective MRLs. So dihydrostreptomycin (DHS) replaced spectinomycin (level of detection in milk: 20000 to $40000 \mu \mathrm{g} \mathrm{I}^{-1}$ ). During the validation of the STAR protocol in milk (Gaudin et al. 2004), the level of detection of colistin was included between 200 and 2000 times its MRL (MRL $50 \mathrm{\mu g} \mathrm{I}^{-1}$ in milk and $150 \mu \mathrm{g} \mathrm{I}^{-1}$ in muscle). Therefore the STAR protocol is not fitted to the detection of colistin or of spectinomycin.

Some antibiotics are frequently detected in routine monitoring samples: the tetracyclines, particularly oxytetracycline (OTC). Thus, tetracycline has been replaced by oxytetracycline and doxycycline was added instead of flumequine. During the validation of the STAR protocol in milk, the limits of detection for OTC and TTC were both equal to $250 \mathrm{\mu g} \mathrm{I}^{-1}$ (Gaudin et al. 2004). The detection limits of doxycyline (50 $\mathrm{mg}$ $\left.\mathrm{I}^{-1}\right)$ and CTC $\left(75 \mathrm{\mu g} \mathrm{I}^{-1}\right)$ were better. Therefore tetracycline family has been divided in 2 groups from which OTC (the least detected) and doxycycline (the best detected) were chosen as representative compounds. 
672 In a similar way, penicillin and cloxacillin have been chosen as the representative compound

673 for the penicillin family because penicillin G (LOD $5 \mu \mathrm{g} \mathrm{I}^{-1}$ ) was the best detected penicillin 674 during the validation of the STAR protocol in milk and cloxacillin (LOD $60 \mu \mathrm{g} \mathrm{I}^{-1}$ ) was the least 675 detected penicillin (Gaudin et al. 2004). Moreover they belong to 2 groups of MRL (penicillin $676 \mathrm{G} 50 \mu \mathrm{g} \mathrm{kg}^{-1}$ in muscle and cloxacillin $300 \mu \mathrm{g} \mathrm{kg}^{-1}$ ). Similarly, erythromycin (LOD $30 \mu \mathrm{I} \mathrm{I}^{-1}$ ) 677 and tilmicosin (LOD $50 \mathrm{\mu g} \mathrm{I}^{-1}$ ) were the best detected macrolides in milk. On the contrary, 678 tylosin (LOD $200 \mu \mathrm{g} \mathrm{I}^{-1}$ ) was the least detected and was representative of the group of 679 spiramycin (LOD $300 \mathrm{\mu g} \mathrm{l}^{-1}$ ) and neospiramycin (LOD $200 \mathrm{\mu g} \mathrm{l}^{-1}$ ). Moreover they also belongs 680 to 2 groups of MRL (tylosin $100 \mu \mathrm{g} \mathrm{kg}^{-1}$ and erythromycin $200 \mu \mathrm{g} \mathrm{kg}^{-1}$ ). Concerning 681 quinolones, enrofloxacin was considered as representative of the quinolones family, except 682 flumequine. In fact, flumequine is usually badly detected by many microbiological methods. 683 When the STAR protocol was validated in milk, we have observed that enrofloxacin 684 (detection limit $\left.=20 \mu \mathrm{g} \mathrm{l}^{-1}\right)$, ciprofloxacin $\left(10 \mu \mathrm{g} \mathrm{I}^{-1}\right)$, marbofloxacin $\left(30 \mu \mathrm{g} \mathrm{I}^{-1}\right.$ ) and 685 danofloxacin $\left(15 \mathrm{\mu g}^{-1}\right)$ had the same activity profiles, with similar sensitivities (Gaudin et al. 686 2004). The quinolones which was badly detected was flumequine $\left(>600 \mathrm{\mu g}^{-1}\right)$. During the 687 validation in milk, sulfanilamide, sulfapyridine, sulfadoxine, sulfacetamide, sulfaquanidine 688 were the least sensitive sulphonamides (3.5 to 20 times the $M R L\left(M R L=100 \mu \mathrm{g} / \mathrm{l}^{-1}\right.$ ) and the 689 most sensitive sulphonamides were sulfaphenazole, sulfathiazole, sulfachloropyridazine 690 (MRL). The limits of detection of sulfadimethoxine (SDMX) (1.75 times the MRL) and 691 sulfamethazine (2.5 times the MRL) were in between. The choice of sulphonamide was not 692 based on the least detected compounds because it was too far from the MRL but was 693 focused on 2 antibiotics which had intermediary sensitivities. Moreover these 2 694 sulphonamides are commonly used for animal treatment. Concerning the aminoglycosides, 695 gentamicin in milk was the best detected antibiotic (limit of detection $300 \mathrm{\mu g} \mathrm{I}^{-1}$ ) and 696 spectinomycin was the least detected aminoglycoside (20000 to $40000 \mathrm{\mu g} \mathrm{l}^{-1}$ ). The limit of 697 detection for neomycin, kanamycin, streptomycin and DHS was equal to $1000 \mu \mathrm{g} \mathrm{I^{-1 }}$. 698 Therefore DHS is representative of the 3 other aminoglycosides. Lincomycin was detected at $699350 \mu \mathrm{g} \mathrm{I}^{-1}$ and pirlimycin at $100 \mu \mathrm{g} \mathrm{I}^{-1}$. Therefore lincomycin was the least detected and is 700 representative of the detection of lincosamides.

702 The specificity of the STAR protocol for the detection of antimicrobial residues in porcine 703 muscle was very satisfactory. A similar result was obtained by Pikkemaat et al. (2009a) when 704 implementing the STAR protocol on routine monitoring muscle samples in a comparative 705 study. Only $1 \%$ of false positive results were observed (6/591), on plate Bst, after the 706 analyses of 591 routine monitoring samples, when the cut-off was set at $4 \mathrm{~mm}$, which is the 707 recommended cut-off in the STAR protocol for the plate Bst. 


\section{1}

708 The results were obviously similar to the global orientation given in the STAR protocol: beta709 lactams and sulphonamides were detected preferentially on plate Bst, tetracyclines on plate $710 \mathrm{Bc} 6$, quinolones on plate $\mathrm{Ec} 8$ and macrolides on plate Kv8. However, there were 2 711 exceptions. The aminoglycosides have been detected on plate Bst with the concentrations 712 tested for the determination of $C C \beta$, whereas for higher concentrations than $C C \beta$, they were 713 detected on plate Bs8 (= Bs7.2). However, when the validation of the STAR protocol has 714

736 The detection capabilities have been determined for 16 antibiotics from different families in 737 relation to their respective Maximum Residue Limit (MRL). The levels of detection of beta738 lactams (penicillins and cephalosporins $\leq$ MRL) were very satisfactory because even the 739 least detected compound has been detected at or below MRL. Concerning tetracyclines, 740 OTC (the least detected) could be detected at levels below 2.5 times the MRL and 741 doxycycline (the best detected) at the MRL. The levels of detection of macrolides (2 MRL), 742 quinolones ( $\leq 2 \mathrm{MRL}$ ) and trimethoprim (2 MRL) were also satisfactory even for the least 743 detected compound. The levels of detection of one representative sulphonamide SDMX $(\leq 3$ 744 MRL) were slightly higher than in milk, Finally, the sensitivity of the STAR protocol towards 
745 aminoglycosides and florfenicol was not satisfactory ( $>>M R L)$. Therefore the interest of the 746 plate Bs8 in the STAR protocol could be discussed. This plate should be improved for its 747 sensitivity or should be replaced by another plate. Other possibility is to use a 748 complementary method focused on aminoglycosides which could replace the use of this fifth 749 plate.

750

751 The validation of microbiological plate test is also a complex issue because of the solid 752 matrices like muscle and kidney. In fact, the ideal solution would be to produce incurred 753 samples from animal treatment for all antibiotics having MRL in the corresponding matrix and 754 to validate all these antibiotics. However this is extremely time consuming and expensive. 755 Furthermore, it is really difficult to obtain exactly a target concentration after the treatment of 756 animals. So there are different possibilities to implement the validation of a microbiological 757 plate test.

759 The first option is to use spiked paper discs to determine the sensitivity of the method from 760 antibiotic standard solutions (Koenen-Dierick et al. 1995; Calderon et al. 1996, Currie et al. 761 1998; Ferrini et al. 2006). The main advantages are that this solution is less expensive and 762 quicker. The validation could be implemented for a wide list of antibiotic residues. However, 763 matrix components could affect the detection capabilities of a method. Okerman et al. 764 (1998a) showed that only tetracyclines and quinolones were similarly detected with or 765 without tissue. However, the detection of beta-lactams was better with antibiotic spiked discs 766 without tissue than with tissues. For some antibiotics, the difference was only observed at 767 low concentrations. Therefore, the use of antibiotic spiked discs is not totally satisfactory.

768 The second possibility is to use monitoring routine samples (Okerman et al. 1998b; 769 Pikkemaat et al. 2009a; Schneider et al. 2009). Routine samples from monitoring plans have 770 been analyzed with different methods including the method to be validated (including one or 771 several physico-chemical confirmatory methods). The first disadvantage is that the number of 772 samples containing residues is unknown. Moreover, very powerful confirmatory methods are 773 needed to confirm all the samples, preferably even negative results at the screening step to 774 check the false negative rate of the screening test. Therefore it is also costly and time 775 consuming. Finally only a narrow range of antibiotics (those which are most frequently used 776 in the country) is encountered. Schneider et al. (2009) confirmed the presence of antibiotics 777 in 29 samples, from which 23 belonged to the tetracycline family. This kind of validation led 778 very interesting information on the method, its practicability in routine conditions and some 779 information on its performance but the results are limited by the range of antibiotics and their 780 concentrations. 


\section{1}

781 Sometimes, the validation study combined spiked discs and analyses of incurred materials, 782 resulting from animal treatment (Myllyniemi et al. 1999; Myllyniemi et al. 2001). In this case, 783 the authors used the activity patterns of antibiotic standard solutions to identify antibiotic 784 residues in incurred materials. Furthermore, the STAR protocol has been validated in our lab 785 using spiked paper discs, plus analysing field routine samples. The interest of spiked discs is 786 787 788 789 790 791 792 793 794 795 796 797 798

799 The third possibility is the use of "simulated tissues" as it is proposed in this paper. It seemed 800 to be a good compromise between spiked discs and incurred tissues and the nearest 801 preparation from intact muscles because matrix effect could be evaluated. For the validation 802 of a microbial screening assay, Pikkemaat et al. (2007) used 2 different approaches to 803 produce fortified tissues. Firstly spiked minced was heated and centrifuged to extract meat 804 juice (supernatant) which was applied directly onto the plate. Secondly incurred tissues were 805 transformed into powder (using liquid nitrogen and blending the meat) and meat juice was 806 extracted. Therefore, it was possible to obtain accurate concentrations in the samples by 807 mixing incurred and blank materials. One conclusion was that "the sensitivity of the assay for 808 quinolones decreased 2 fold when matrix samples are analysed" by comparison with 809 antibiotic standard solutions. In these conditions, matrix effect was taken into account. 810 Moreover, these 2 approaches are fitted for this screening test because in routine, meat juice 811 samples would be analysed also. However, that sample preparation differed a lot from the 812 routine application of the STAR protocol which is based on pieces of raw muscles laid 813 directly on the plates. Therefore, in our case, our "simulated tissues" appeared to be as near 814 as possible from real muscle samples. The binding of antibiotics to the minced tissue is 815 possible during spiking. In 2009, Pikkemaat et al validated the NAT-post-screening test for 816 the detection of antibiotic residues in kidney by determining the detection capabilities of 36 817 antibiotics in porcine as well as in homogenised fortified kidney samples (Pikkemaat et al. 
818 2009b). After centrifugation, the supernatant could was analysed. The same final treatment

819 was applied to routine monitoring samples. This is another way of preparing simulated 820 tissues.

822 In the decision EC/2002/657 (EC 2002), there is no recommendation concerning the kind of 823 materials to be analysed (spiked or incurred matrix) during the validation. Therefore all of the 824 previous validation studies could be in accordance with the European decision if the way of 825 determining performance characteristics was respected. The most important thing is to keep 826 as close as possible to the materials analysed in routine use by the concerned method. Now 827 the guideline for validation of screening methods (Anonymous 2010) recommends the use of 828 "simulated tissues" (spiked tissues) when it concerns solid matrices and when it is impossible 829 to obtain incurred tissues. Moreover, the validation could be restricted to a list of 830 representative antibiotics. The validation which have been performed in the past on field 831 samples led very interesting information concerning the method. However this kind of 832 validation does not fit to the decision $\mathrm{EC} / 2002 / 657$ and to the recommendations of the 833 validation guideline (Anonymous 2010). In fact the number of antibiotics tested is always very 834 restricted. Moreover the number of samples to be analysed to determine the detection 835 capabilities of antibiotics is never respected.

836

837 As a conclusion, there is no ideal way of validating microbiological plate tests which would 838 not be time and money consuming. Obviously, validation of these kind of methods needs a 839 lot of efforts from the lab which would validate the protocol for the first time (initial validation). 840 In the guideline for validation of screening methods (Anonymous 2010), one of the 841 recommendations is to reduce the work of validation when the method is transferred to 842 another laboratory which would want to implement it in routine conditions. In fact, the number 843 of samples to be analysed is reduced. The performance characteristics (e.g. detection 844 capabilities) determined in the transfer lab have to be compared to those determined during 845 the initial validation for a selected range of antibiotics. Then the participation to proficiency 846 testing studies could complete the validation dossier.

\section{Conclusions}

849 The two objectives of this validation study have been fulfilled. Firstly, the STAR protocol has 850 been validated for muscle by determining performance characteristics (specificity, detection 851 capabilities $C C \beta$ for 16 representative antimicrobials, applicability, ruggedness), according to 852 the European decision 2002/657/EC (EC 2002). The STAR protocol is applicable to the 853 broad spectrum detection of antibiotic residues in muscles of different animal species (pig, 854 cattle, sheep, poultry.). The method has good specificity (false positive rate 4\%). The 
855 detection capabilities have been determined for 16 antibiotics from different families in 856 relation to their respective Maximum Residue Limit (MRL): beta-lactams (penicillins and 857 cephalosporins $\leq \mathrm{MRL}$ ), tetracyclines ( $\leq \mathrm{MRL}$ and $\leq 2.5 \mathrm{MRL}$ ), macrolides (2 $\mathrm{MRL}$ ), 858 quinolones $(\leq 2 \mathrm{MRL})$, some sulphonamides ( $\leq 3 \mathrm{MRL}$ ), trimethoprim (2MRL). However, the 859 sensitivity of the STAR protocol towards aminoglycosides and florfenicol was not satisfactory 860 (>>MRL). Finally, this study has shown that the STAR method is a robust screening method, 861 insensitive to reasonable variations analytical parameters such as concentration of bacteria, 862 amount of medium, incubation period and pre-incubation or not. 863

864 Secondly, the applicability of the European guideline (Anonymous 2010) to the validation of a 865 microbiological screening method and for muscle has been demonstrated. The use of 866 "simulated tissue" appeared to be a very good compromise between antibiotic spiked discs 867 and incurred samples. Moreover, the choice of a list of representative antimicrobials was also 868 very interesting to reduce the scope of validation.

869

\section{REFERENCES}

871 Anonymous. 2010. Document of $21^{\text {st }}$ of January 2010. Guidelines for the validation of 872 screening methods for residues of veterinary medicines (Initial validation and transfer of 873 method).

874 Calderon V, Gonzalez J, Diez P, Berenguer JA. 1996. Evaluation of a multiple bioassay 875 technique for determination of antibiotic residues in meat with standard solutions of 876 antimicrobials. Food Additives and Contaminants. 13:13-19.

877 Currie D, Lynas L, Kennedy DG, McCaughey WJ. 1998. Evaluation of a modified EC Four 878 Plate Method to detect antimicrobial drugs. Food Additives and Contaminants. 15:651879660.

880 EC 1990. Council Regulation (EEC) No. 2377/90. 26 June 1990. Laying down a Community 881 procedure for the establishment of maximum residue limits of veterinary medicinal 882 products in foodstuffs of animal origin. Official Journal of the European Community. 883 L224:1-8.

884 EC 1996. Council Directive (EEC) No. 96/23/EC of 29 April 1996 on measures to monitor 885 certain substances and residues thereof in live animals and animal products. Official 886 Journal of the European Community. L125:10-32.

887 EC 2002. Commission Decision 2002/657/EC of 12 August 2002 implementing Council 888 Directive 96/23/EC concerning the performance of analytical methods and interpretation of 889 results. Official Journal of the European Community. L 221: 8-36. 
890 EC 2009. Council Regulation (EEC) No 470/2009 of 6 May 2009 laying down Community 891 procedures for the establishment of residue limits of pharmacologically active substances 892 in foodstuffs of animal origin, repealing Council Regulation (EEC) No 2377/90. Official 893 Journal of the European Community. L152:11-22.

894 Ferrini AM, Mannoni V, Aureli P. 2006. Combined Plate Microbial Assay (CPMA): A 6-plate895 method for simultaneous first and second level screening of antibacterial residues in meat. 896 Food Additives and Contaminants. 23:16-24.

897 Fuselier R, Cadieu N, Maris P, in: L.A. van Ginkel, A. Ruiter (Eds.), proceedings of the 898 Euroresidue IV Conference, National Institute of Public Health (RVM), Bilthoven, 2000, p. 899444.

900 Gaudin V, Maris P, Fuselier R, Ribouchon JL, Cadieu N, Rault A. 2004. Validation of a 901 microbiological method: the STAR protocol, a five-plate test, for the screening of antibiotic 902 residues in milk. Food Additives and Contaminants. 21: 422-433.

903 Gaudin V, Hedou C, Sanders P. 2007. Validation of a Biacore Method for Screening Eight 904 Sulfonamides in Milk and Porcine Muscle Tissues According to European Decision 905 2002/657/EC. JOURNAL OF AOAC International. 90 (6):1706-1715.

906 Gaudin V, Hedou C, Rault A, Sanders P, Verdon E. 2009a. Comparative study of 3 907 screening tests, Explorer ${ }^{\circledR}$ test, PremißTest 2 microbiological tube tests and a multi908 sulphonamides ELISA kit, for the detection of antimicrobial and sulphonamide residues in 909

910 Gaudin V, Hedou C, Verdon E. 2009b. Validation of a wide-spectrum microbiological tube 911 test, the EXPLORER® test, for the detection of antimicrobials in muscle from different 912 animal species. Food Additives \& Contaminants: Part A. 26 (8) 1162-1171.

913 Koenen-Dierick K, Okerman L, De Zutter L, Degroodt JM, Van Hoof J, Srebnik S. 1995. A 914 one-plate microbiological screening test for antibiotic residue testing in kidney tissue and 915 meat : an alternative to the EEC four-plate method?. Food Additives and Contaminants. 916 12:77-82.

917 Myllyniemi, A.-L., Rintala, R., Backman, C., and Niemi, A., 1999, Microbiological and 918 chemical identification of antimicrobial drugs in kidney and muscle samples of bovine 919 cattle and pigs. Food Additives and Contaminants. 16:339-351.

920 Myllyniemi AL, Nuotio L, Lindfors E, Rannikko R, Niemi A, Backmann C. 2001. A 921 microbiological six-plate method for the identification of certain antibiotic groups in 922 incurred kidney and muscle samples. Analyst 126:641-646. 
923 Okerman L, De Wasch K and Van Hoof J. 1998a. Detection of antibiotics in muscle tissue 924 with microbiological inhibition tests: effects of the matrix. Analyst. 123:2361-2365.

925 Okerman L, Van Hoof J, Debeuckelaere W. 1998b. Evaluation of the European four-plate 926 test as a tool for screening antibiotic residues in meat samples from retail outlets. Journal 927 of AOAC International. 81:51-56.

928 Okerman L, Croubels S, Cherlet M, De Wasch K, De Backer P, Van Hoof J. 2004. Evaluation 929 and establishing the performance of different screening tests for tetracycline residues in 930 animal tissues. Food Additives and Contaminants. 21:145-153.

931 Pikkemaat MG, Mulder PPJ, Elferink JWA., De Cocq A, Nielen MWF., Van Egmond HJ. 932 2007. Improved microbial screening assay for the detection of quinolone residues in 933 poultry and eggs. Food Additives and Contaminants. 24(8):842-850.

934 Pikkemaat MG, Oostra-van Dijk S, Schouten J, Rapallini M, van Egmond HJ. 2008. A new 935 microbial screening method for the detection of antimicrobial residues in slaughter 936 animals: The Nouws antibiotic test (NAT-screening). Food Control 19:781-789

937 Pikkemaat MG, Rapallini Michel L.B.A., Oostra-van Dijk Sabrina, Elferink J.W. Alexander. 938 2009a. Comparison of three microbial screening methods for antibiotics using routine 939 monitoring samples. Analytica Chimica Acta. 637:298-304.

940 Pikkemaat MG, Oostra-van Dijk S, Schouten J, Rapallini M, Kortenhoeven L, van Egmond 941 HJ. 2009b. Nouws antibiotic test: Validation of a post-screening method for antibiotic 942 residues in kidney. Food Control. 20:771-777

943 Renard L, Moulin G, Sanders P. 1992. Using experimental design to optimize a diffusion 944 assay. Journal of AOAC International. 75 (6):1045-1048.

945 Schneider MJ, Mastovska K, Lehotay, Lightfield A, Kinsella, Shultz C. 2009. Comparison of 946 screening methods for antibiotics in beef kidney juice and serum. Analytica Chimica Acta. $947 \quad 637: 290-297$. 


\section{Figure 1. Preparation of the simulated tissues.}

949 Two technicians prepared each 30 samples per antibiotic (AB), divided in 2 sets of 15 950 samples each (Set 1 and Set 2). The 2 sets have been spaced out, to introduce variability in 951 the period of analysis (ambient conditions) and therefore variability of batches (media, 952 bacteria, antibiotic standard...). The example of preparation of Set 1 by technician 1 is 953 presented here. Four different batches of porcine muscle have been used. Two different 954 working solutions (WS) have been prepared from the stock solution of antibiotic and used to 955 spike the different batches of muscle. Finally 15 samples have been prepared per antibiotic. 956 957

958 Figure 2. Relationship between the calculated detection capabilities and the respective 959 MRLs of the 16 representative antibiotics.

960 The 16 representative antibiotics are represented in $x$-axis. The calculated CC $\beta$ of the 16 961 tested representative antimicrobials and the respective MRLs are represented in $y$-axis. MRL 962 = Maximum Residue Limit. 963 964

965 Figure 3. Results of the applicability study of the STAR protocol to muscles from 966 different animal species, on the 5 different plates.

967 The results of the applicability study for the 5 plates are represented: $2 a$. penicillin $\mathrm{G}$ at $25 \mu \mathrm{g}$ $968 \mathrm{~kg}^{-1}$ on plate Bst, 2b. doxycycline at $100 \mathrm{\mu g} \mathrm{kg}^{-1}$ on Plate Bc6, 2c. erythromycin at $400 \mu \mathrm{gg}^{-1}$ 969 on Plate $\mathrm{Kv8}, 2 \mathrm{~d}$. enrofloxacin of $200 \mu \mathrm{g} \mathrm{kg}^{-1}$ on Plate Ec8 and 2e. gentamicin at $6000 \mu \mathrm{g} \mathrm{kg}$ $970{ }^{1}$ on Plate Bs8. In $x$-axis, the 4 different animal species tested are represented. In $y$-axis, the 971 average inhibition zone (in $\mathrm{mm}$ ) on the 5 different plates obtained during the applicability 972 study for bovine, ovine and poultry muscles and during the determination of CC $\beta$ for porcine 973 muscle are represented for blank samples and spiked samples. 
Figure 1.
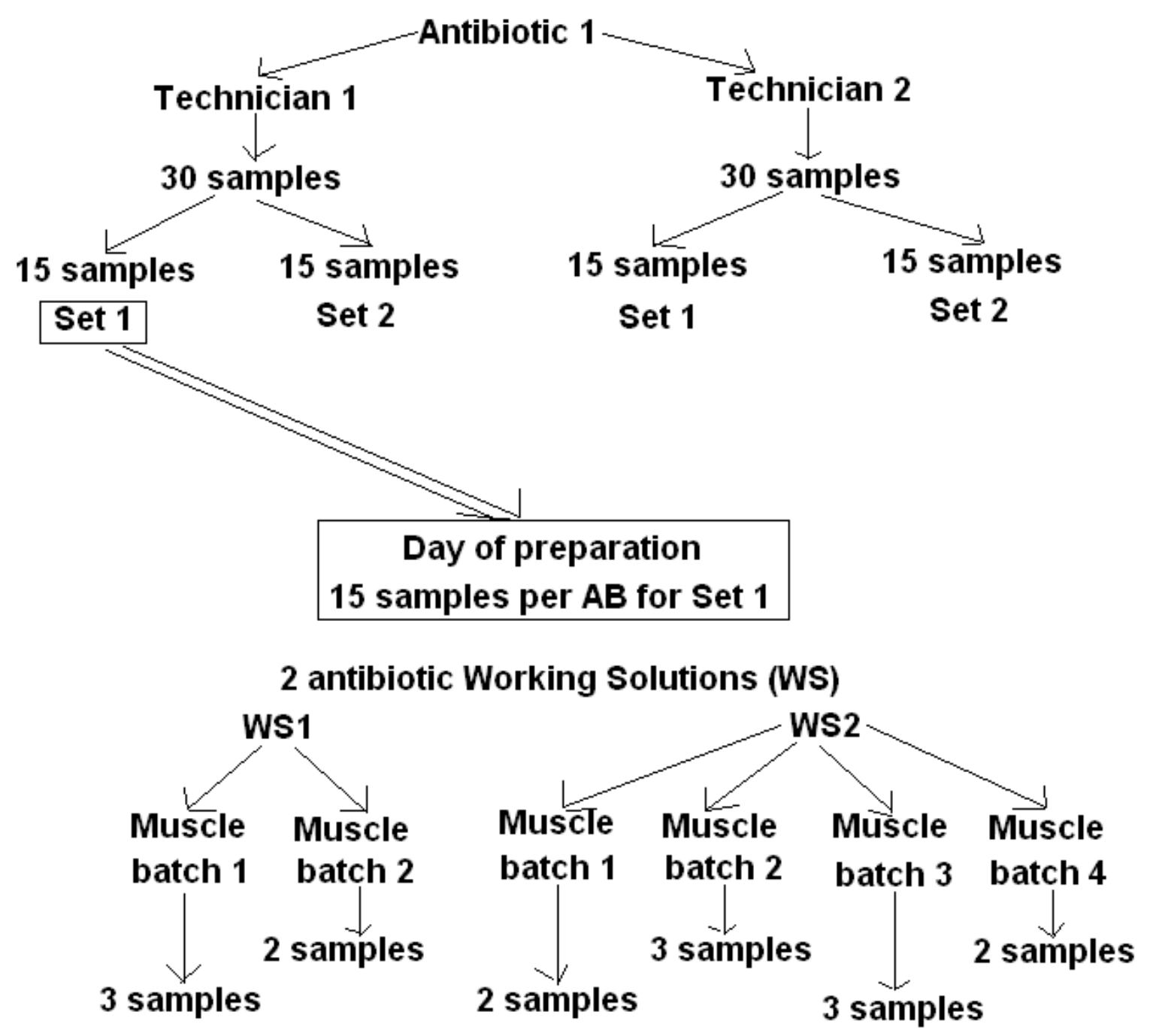
Figure 2.

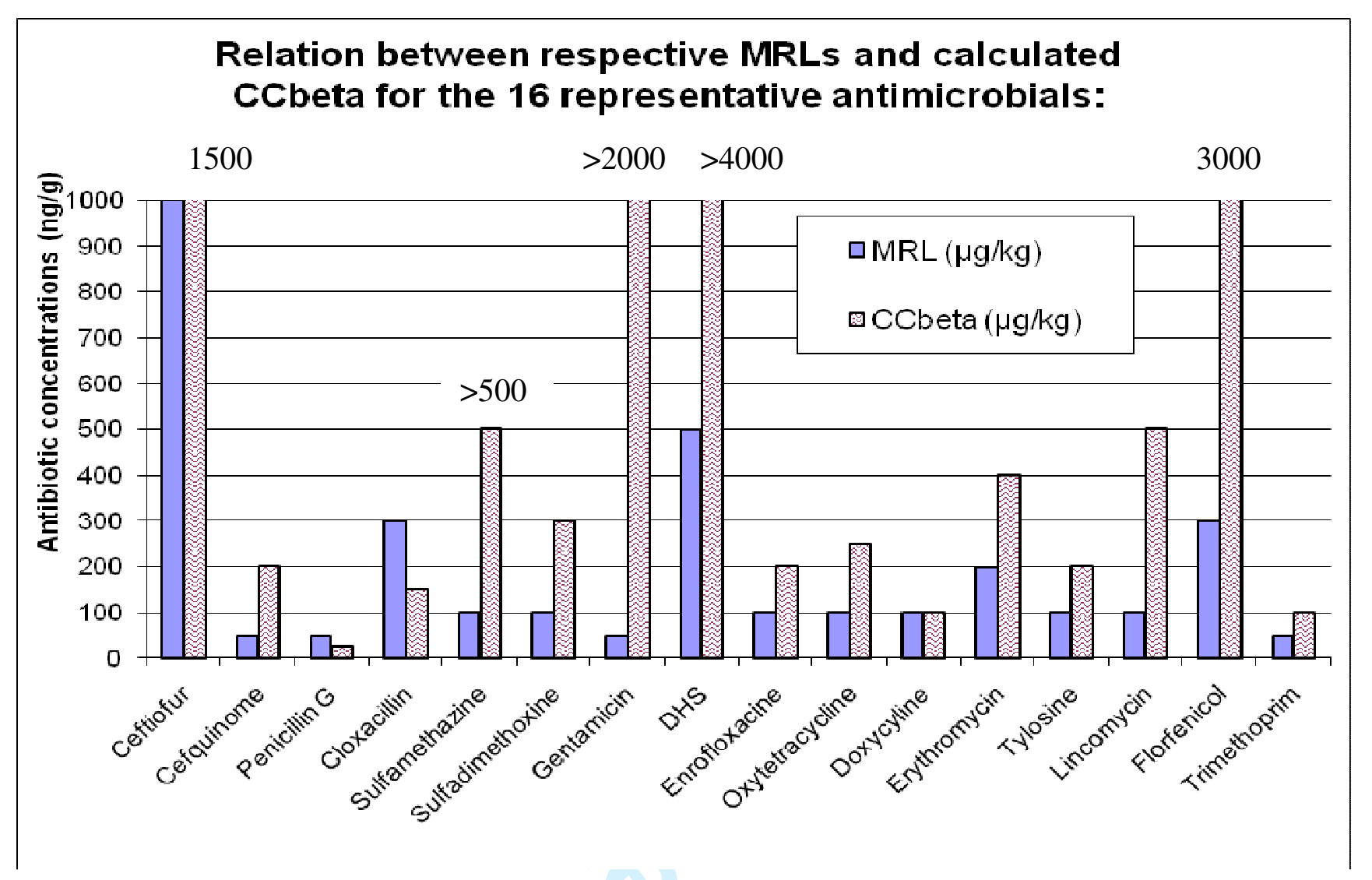


Figure 3.

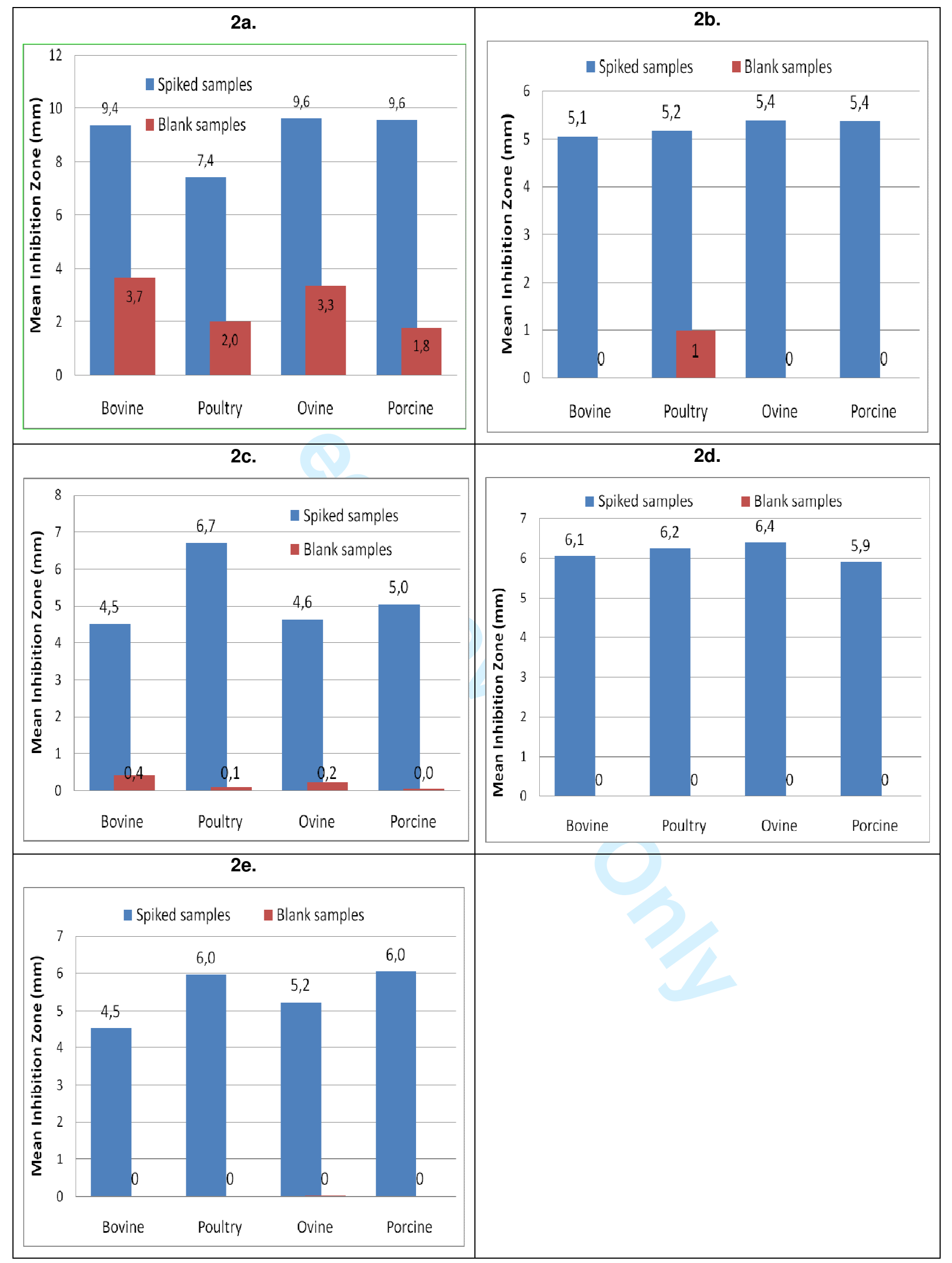


Table 1. Chosen antibiotics and corresponding concentrations for the validation study.

\begin{tabular}{|c|c|c|c|}
\hline Antibiotic families & Representative antibiotics & $\underset{\left.\mathbf{k g}^{-1}\right)}{\operatorname{MRL}(\mu \mathrm{g}}$ & $\begin{array}{c}\text { Chosen } \\
\text { concentrations } \\
\left(\mu \mathrm{g} \mathrm{kg}^{-1}\right)\end{array}$ \\
\hline CEPHALOSPORINS & Ceftiofur/Cefquinome & $1000 / 50$ & $1500 / 200$ \\
\hline PENICILLINS & Penicillin G/Cloxacillin & $50 / 300$ & $25 / 150$ \\
\hline SULFONAMIDES & Sulfamethazine/Sulfadimethoxine & $100 / 100$ & $500 / 300$ \\
\hline AMINOGLYCOSIDES & $\begin{array}{c}\text { Gentamicin (GTM) } \\
\text { /Dihydrostreptomycin (DHS) }\end{array}$ & $50 / 500$ & $2000 / 4000$ \\
\hline QUINOLONES & Enrofloxacin & 100 & 200 \\
\hline TETRACYCLINES & Oxytetracycline/Doxycycline & $100 / 100$ & $250 / 100$ \\
\hline MACROLIDES & Erythromycin/Tylosin & $200 / 100$ & $400 / 200$ \\
\hline LINCOSAMIDES & Lincomycin & 100 & 500 \\
\hline PHENICOLS & Florfenicol & 300 & 3000 \\
\hline MISCELLANEOUS & Trimethoprim & 50 & 100 \\
\hline
\end{tabular}

The antibiotics in italics are the antibiotics which have been substituted to the antibiotics initially included in the list of representative antibiotics. 
Table 2. Design matrixof the ruggedness study .

\begin{tabular}{|c|c|c|c|c|c|c|c|c|}
\hline Run & Levels & $A^{a}$ & $\mathbf{B}^{b}$ & $\mathrm{C}^{\mathrm{C}}$ & $D=A B C^{d}$ & $A B+C D$ & $A C+B D$ & $B C+A D$ \\
\hline 1 & + & $-^{*}$ & - & - & - & + & + & + \\
\hline 2 & + & + & - & - & + & - & - & + \\
\hline 3 & + & - & + & - & + & - & + & - \\
\hline 4 & + & + & + & - & - & + & - & - \\
\hline 5 & + & - & - & + & + & + & - & - \\
\hline 6 & + & + & - & + & - & - & + & - \\
\hline 7 & + & - & + & + & - & - & - & + \\
\hline 8 & + & + & + & + & + & + & + & + \\
\hline
\end{tabular}

Run $1=$ Day 1

${ }^{a}$ Concentration of bacteria; ${ }^{b}$ Medium quantity; ${ }^{c}$ Incubation time, ${ }^{d}$ Pre-incubation time

* Each factor has been modified in an order of magnitude corresponding to the usual differences: factor $A$ concentration in bacteria: $\pm 30 \%$; factor $B$ quantity of medium $5 \pm 0.5 \mathrm{ml}$; factor $C$ incubation time $\pm 10 \%$; factor $D$ pre-incubation time (period at room temperature before incubation in the incubator: 1hour of preincubation or no pre-incubation).

+: increasing of the factor (i.e. $+30 \%$ ); -: decreasing of the factor (i.e. - $30 \%$ )

$A B+C D, A C+B D$ and $B C+A D$ are the evaluation of the impact of the combination of the different factors 
Table 3. Detection capabilities $C C \beta\left(\mu \mathrm{g} \mathrm{kg}^{-1}\right.$ ) for the 16 antimicrobials

\begin{tabular}{|c|c|c|c|c|c|}
\hline Antibiotic family & Antibiotic & $\begin{array}{c}\text { MRL } \\
\text { muscle } \\
\left(\mu \mathrm{g} \mathrm{kg}^{-1}\right)\end{array}$ & $\begin{array}{l}\text { Mean IZ * } \\
(\mathrm{mm}) \pm \\
(\mathrm{SD})\end{array}$ & Qualitative results & $\begin{array}{c}C C \beta \\
\left(\mu \mathrm{kg}^{-1}\right)\end{array}$ \\
\hline \multirow[b]{2}{*}{ PENICILLINS } & Penicillin G & 50 & $9.7 \pm 1.1$ & $60+$ & $\leq 25$ \\
\hline & Cloxacillin & 300 & $6.4 \pm 0.2$ & $\begin{array}{c}56+/ 4- \\
\left(\text { at } 150 \mu \mathrm{g} \mathrm{kg}^{-1} \text { ) }\right.\end{array}$ & $\begin{array}{c}\text { Between } 150 \\
\text { and } 300\end{array}$ \\
\hline \multirow[t]{2}{*}{ CEPHALOSPORINS } & Cefquinome & 50 & $6.0 \pm 0.3$ & $\begin{array}{c}\left.\text { 14+/1D (at } 100 \mu \mathrm{g} \mathrm{kg}^{-1}\right) \\
60+\left(\text { at } 200 \mu \mathrm{gg}^{-1}\right)\end{array}$ & $\begin{array}{c}\text { Between } 100 \\
\text { and } 200\end{array}$ \\
\hline & Ceftiofur & 1000 & $5.3 \pm 0.7$ & $56+/ 2 D / 2-$ & 1500 \\
\hline \multirow[t]{2}{*}{ TETRACYCLINES } & $\begin{array}{l}\text { Oxytetracycline } \\
\text { (OTC) }\end{array}$ & 100 & $5.1 \pm 0.5$ & $60+$ & $\leq 250$ \\
\hline & Doxycycline & 100 & $5.4 \pm 0.5$ & $60+$ & $\leq 100$ \\
\hline \multirow{2}{*}{ MACROLIDES } & Erythromycin & 200 & $5.0 \pm 1.6$ & $56+/ 1 \mathrm{D} / 3-$ & 400 \\
\hline & Tylosin & 100 & $5.1 \pm 0.7$ & $55+/ 3 D / 2-$ & 200 \\
\hline QUINOLONES & Enrofloxacin & 100 & $5.9 \pm 1.9$ & $\begin{array}{c}8+/ 4 \mathrm{D} / 3-\left(\text { at } 100 \mu \mathrm{g} \mathrm{kg}^{-1}\right) \\
60+\left(\text { at } 200 \mu \mathrm{g} \mathrm{kg}^{-1}\right)\end{array}$ & $\begin{array}{c}\text { Between } 100 \\
\text { and } 200\end{array}$ \\
\hline \multirow{2}{*}{ SULFONAMIDES } & $\begin{array}{l}\text { Sulfadimethoxin } \\
\text { e (SDMX) }\end{array}$ & 100 & $8.8 \pm 1.3$ & $\begin{array}{c}\left.\text { 12+/1D/2- (at } 200 \mu \mathrm{g} \mathrm{kg}^{-1}\right) \\
59+/ 1 \mathrm{D}\left(\text { at } 300 \mu \mathrm{gg}^{-1}\right)\end{array}$ & $\begin{array}{c}\text { Between } 200 \\
\text { and } 300\end{array}$ \\
\hline & $\begin{array}{l}\text { Sulfamethazine } \\
\text { (SMZ) }\end{array}$ & 100 & $5.4 \pm 2.5$ & $37+/ 2 D / 21-$ & $>500$ \\
\hline \multirow{2}{*}{$\begin{array}{c}\text { AMINOGLYCOSIDE } \\
\text { S }\end{array}$} & $\begin{array}{c}\text { Dihydrostreptom } \\
\text { ycin (DHS) }\end{array}$ & 500 & $2.0 \pm 0.7$ & $24+/ 3 D / 33-$ & $>4000$ \\
\hline & $\begin{array}{l}\text { Gentamicin } \\
\text { (GTM) }\end{array}$ & 50 & $4.3 \pm 0.4$ & $45+/ 3 D / 12-$ & $>2000$ \\
\hline LINCOSAMIDES & Lincomycin & 100 & $5.8 \pm 0.6$ & $60+\left(\right.$ at $\left.500 \mu \mathrm{g} \mathrm{kg}^{-1}\right)$ & $\begin{array}{c}\text { Between } 350 \\
\text { and } 500\end{array}$ \\
\hline \multirow{2}{*}{ MISCELLANEOUS } & $\begin{array}{l}\text { Trimethoprim } \\
\text { (TMP) }\end{array}$ & 50 & $7.9 \pm 0.8$ & $\begin{array}{c}5+/ 10-\left(\text { at } 50 \mu \mathrm{g} \mathrm{kg}^{-1}\right) \\
59+\text { out of } 59 \\
\left(\text { at } 100 \mu \mathrm{gg}^{-1}\right)\end{array}$ & $\begin{array}{c}\text { Between } 50 \\
\text { and } 100\end{array}$ \\
\hline & Florfenicol & 300 & $4.8 \pm 0.2$ & $\begin{array}{c}8+/ 3 \mathrm{D} / 19-\left(\text { at } 1000 \mu \mathrm{g} \mathrm{kg}^{-1}\right) \\
60+\left(\text { at } 3000 \mu \mathrm{g} \mathrm{kg}^{-1}\right)\end{array}$ & $\begin{array}{c}\text { Between } \\
1000 \text { and } \\
3000\end{array}$ \\
\hline
\end{tabular}

$S D$ = Standard deviation; IZ: Inhibition $\overline{\text { zone }(\mathrm{mm})} ;$ +: positive result; -: negative result; $D$ : doubtful result $(0<Z \mathrm{l}<2 \mathrm{~mm})$. 
Table 4. Specificity of the plates.

\begin{tabular}{|c|c|c|}
\hline Antibiotic family & Antibiotic & Specific plate \\
\hline \multirow{2}{*}{ PENICILLINS } & Penicillin G & Bst \\
\hline & Cloxacilline & Bst \\
\hline \multirow{2}{*}{ CEPHALOSPORINES } & Cefquinome & Kv8 (Bst and Ec8) \\
\hline & Ceftiofur & Bst (and Ec8) \\
\hline \multirow{2}{*}{ TETRACYCLINES } & Oxytetracycline (OTC) & $\mathrm{Bc6}$ \\
\hline & Doxycycline & Bc6 (and Bs8) \\
\hline \multirow{2}{*}{ MACROLIDES } & Erythromycin & Kv8 \\
\hline & Tylosin & Bst \\
\hline QUINOLONES & Enrofloxacin & Ec8 (and Bst) \\
\hline \multirow{2}{*}{ SULFONAMIDES } & Sulfadimethoxine (SDMX) & Bst \\
\hline & Sulfamethazine (SMZ) & Bst \\
\hline \multirow{2}{*}{ AMINOGLYCOSIDES } & Dihydrostreptomycin (DHS) & Bst \\
\hline & Gentamicin (GTM) & Bst \\
\hline LINCOSAMIDES & Lincomycin & Bst \\
\hline \multirow{2}{*}{ MISCELLANEOUS } & Trimethoprim (TMP) & Bst \\
\hline & Florfenicol & Bs8 (Bc6 and Bst) \\
\hline
\end{tabular}


Table 5. Comparison of the mean Inhibition zones (IZ) and the standard deviations (SD) calculated during the determination of the $C C \beta$ and during the ruggedness study.

\begin{tabular}{cccccc}
\hline Antibiotic family & Antibiotic & $\begin{array}{c}\text { Tested } \\
\text { concentratio } \\
\mathbf{n}\left(\boldsymbol{\mu \mathbf { ~ k g } ^ { - 1 } )}\right.\end{array}$ & Plate & $\begin{array}{c}\text { Mean IZ }{ }^{*}(\mathbf{m m}) \pm \\
\mathbf{S D} \\
\mathbf{C C} \boldsymbol{\beta}\end{array}$ & $\begin{array}{c}\text { Mean IZ * }(\mathbf{m m}) \pm \\
\text { Ruggedness }\end{array}$ \\
\hline PENICILLINS & Penicillin G & 25 & Bst & $9.7+/-0.2$ & $8.5+/-1.2$ \\
\hline SULFONAMIDES & $\begin{array}{c}\text { Sulfadimethoxine } \\
(\text { SDMX) }\end{array}$ & 300 & Bst & $8.8+/-1.3$ & $5.3+/-1.6$ \\
\hline TETRACYCLINES & Doxycycline & 100 & Bc6 & $5.4+/-0.5$ & $4.3+/-0.6$ \\
\hline MACROLIDES & Erythromycin & 400 & Kv8 & $5.1+/-1.1$ & $4.0+/-1.0$ \\
\hline QUINOLONES & Enrofloxacin & 200 & Ec8 & $6.2+/-1.0$ & $6.7+/-1.2$ \\
\hline AMINOGLYCOSIDES & GTM & 6000 & Bs8 & $6,0+/-0,7$ & $6.2+/-0.5$ \\
\hline
\end{tabular}

${ }^{*} I Z=$ Inhibition zone of the specific plate, mean of the values of the 4 sets (2 technicians)

$S D=$ Standard Deviation 
Table 6. Exploitation of the factorial design : Influence of the 4 experimental factors on the detection of 6 tested antibiotics on the 5 plates of the STAR protocol.

\begin{tabular}{|c|c|c|c|c|c|c|c|c|c|}
\hline & & \multicolumn{4}{|c|}{ Factor } & \multicolumn{3}{|c|}{ Interaction } & \multirow{2}{*}{$\frac{\text { Mean }}{l}$} \\
\hline Bc6 & Response & $\overline{A^{a}}$ & $B^{D}$ & $\overline{C^{C}}$ & $D^{d}=A B C$ & $A B+C D$ & $A C+B D$ & $B C+A D$ & \\
\hline & Mean IZ & -0.12 & -0.01 & -0.15 & 0.05 & -0.13 & 0.01 & -0.16 & 4.32 \\
\hline & CV (\%) & -2.03 & -3.08 & 5.23 & 2.75 & 2.91 & -3.09 & -1.88 & 11.21 \\
\hline \multirow[t]{3}{*}{ Kv8 } & Response & $\bar{A}$ & $\bar{B}$ & $C$ & $D=A B C$ & $A B+C D$ & $A C+B D$ & $B C+A D$ & $T$ \\
\hline & Mean IZ & -0.07 & 0.00 & $0.50^{\star}$ & 0.28 & 0.12 & -0.41 & 0.22 & 3.97 \\
\hline & CV (\%) & -1.27 & 4.04 & 0.58 & 5.43 & 0.07 & 2.69 & -1.93 & 16.07 \\
\hline \multirow[t]{3}{*}{ Ec8 } & Response & $A$ & $B$ & $C$ & $D=A B C$ & $A B+C D$ & $A C+B D$ & $B C+A D$ & $T$ \\
\hline & Mean IZ & -0.25 & -0.58 & -0.22 & 0.77 & -0.45 & -0.03 & -0.33 & 6.72 \\
\hline & CV (\%) & 0.38 & 0.97 & -0.07 & -1.14 & -0.47 & -0.08 & 1.88 & 4.82 \\
\hline Bst & Response & $\bar{A}$ & $B$ & $C$ & $D=A B C$ & $A B+C D$ & $A C+B D$ & $B C+A D$ & $I$ \\
\hline \multirow[t]{4}{*}{ SDMX } & Mean IZ & 0.34 & -0.38 & 0.17 & 0.92 & -0.30 & -0.03 & 0.91 & 5.30 \\
\hline & $\mathrm{CV}$ & -0.26 & 0.15 & -2.72 & -0.01 & 0.25 & -0.76 & 1.70 & 4.70 \\
\hline & False + & 0.00 & 0.00 & 0.00 & 0.00 & 0.00 & 0.00 & 0.00 & 0.00 \\
\hline & False - & 0.50 & 0.50 & -0.50 & -0.50 & 0.50 & -0.50 & -0.50 & 0.50 \\
\hline \multirow[t]{4}{*}{ Penicillin } & Mean IZ & 0.25 & -0.20 & -0.44 & 0.02 & 0.59 & -0.42 & 0.03 & 8.55 \\
\hline & $\mathrm{CV}$ & -1.44 & 1.61 & -2.91 & 1.74 & -2.60 & 3.17 & 0.12 & 9.18 \\
\hline & False + & 0.00 & 0.00 & 0.00 & 0.00 & 0.00 & 0.00 & 0.00 & 0.00 \\
\hline & False - & 0.00 & 0.00 & 0.00 & 0.00 & 0.00 & 0.00 & 0.00 & 0.00 \\
\hline \multirow[t]{3}{*}{ Bs8 } & Response & $A$ & $B$ & $C$ & $D=A B C$ & $A B+C D$ & $A C+B D$ & $B C+A D$ & $T$ \\
\hline & Mean IZ & -0.11 & 0.00 & 0.03 & 0.02 & -0.11 & -0.23 & 0.00 & 6.16 \\
\hline & CV (\%) & 0.67 & -0.84 & 0.65 & 0.53 & 0.01 & 0.14 & 0.81 & 6.07 \\
\hline
\end{tabular}

${ }^{a}$ Concentration of bacteria; ${ }^{b}$ Medium quantity; ${ }^{c}$ Incubation time, ${ }^{d}$ Pre-incubation time

$I Z$ = Inhibition zone $5 \mathrm{MM}^{\circ} ; \mathrm{CV}$ : Coefficient of variation of the mean IZ (\%); False +: False positive rate; False -: False negative rate. The mean false negative rate is equal to 0.50 , that means $50 \%$ of false negative results. * Increasing the incubation time (C) for plate Kv8 increased a little the sensitivity of the plate (13\%). 
Table 7. Ruggedness study for plate Bst: Design matrix and experimental design calculation.

\begin{tabular}{ccccccccc}
\hline Run & $\begin{array}{c}\text { Mean IZ } \\
\text { SDMX }\end{array}$ & CV & False + & False - & $\begin{array}{c}\text { Mean IZ } \\
\text { Penicillin }\end{array}$ & CV & False + & False - \\
\hline $\mathbf{1}$ & 4.8 & 17.5 & 0 & 0 & 9.1 & 10.9 & 0 & 0 \\
\hline $\mathbf{2}$ & 8.0 & 18.0 & 0 & 0 & 9.3 & 10.3 & 0 & 0 \\
\hline $\mathbf{3}$ & 4.7 & 13.9 & 0 & 0 & 7.5 & 22.5 & 0 & 0 \\
\hline $\mathbf{4}$ & 3.0 & 15.4 & 0 & $100^{*}$ & 10.0 & 4.6 & 0 & 0 \\
\hline $\mathbf{5}$ & 5.3 & 10.2 & 0 & 0 & 9.1 & 2.0 & 0 & 0 \\
\hline $\mathbf{6}$ & 4.6 & 7.6 & 0 & 0 & 7.5 & 7.1 & 0 & 0 \\
\hline $\mathbf{7}$ & 5.1 & 13.4 & 0 & 0 & 7.5 & 7.1 & 0 & 0 \\
\hline $\mathbf{8}$ & 6.9 & 11.8 & 0 & 0 & 8.4 & 8.9 & 0 & 0 \\
\hline
\end{tabular}

IZ: Inhibition zone $(\mathrm{mm})$; False $+=$ False positive rate; False $-:$ false negative rate; $\mathrm{CV}$ : Coefficient of variation $(\%)=($ mean $I Z) /(S D I Z)^{*} 100 ; S D$ : standard deviation .

Run $1=$ Day 1

*A strong impact of the combination of factors tested on day 4 was observed on false - rate: $A+, B+$, $C$-, $D$-, i.e. increasing the concentration of bacteria, increasing the amount of medium, reducing the incubation period and without pre-incubation. 